\title{
ESA'S LIDAR MISSIONS AEOLUS AND EARTHCARE
}

\author{
Thomas Kanitz $^{1^{*}}$, Alessandra Ciapponi ${ }^{1}$, Alessia Mondello ${ }^{4}$, Alessandro D'Ottavi ${ }^{4}$, Ana Baselga \\ Mateo $^{1}$, Anne-Grete Straume ${ }^{1}$, Christoph Voland ${ }^{1}$, Didier Bon ${ }^{3}$, Elena Checa ${ }^{1}$, Emilio Alvarez ${ }^{1}$, Ida \\ Bellucci $^{4}$, Joao Pereira Do Carmo ${ }^{1}$, John Brewster ${ }^{2}$, Jon Marshall ${ }^{2}$, Marc Schillinger ${ }^{3}$, Mark \\ Hannington $^{2}$, Michael Rennie ${ }^{7}$, Oliver Reitebuch ${ }^{6}$, Olivier Lecrenier $^{3}$, Paolo Bravetti ${ }^{5}$, Valentina \\ Sacchieri $^{4}$, Valeria De Sanctis ${ }^{4}$, Alain Lefebvre ${ }^{1}$, Tommaso Parrinello ${ }^{8}$, and Denny Wernham ${ }^{1}$ \\ ${ }^{I}$ ESA-ESTEC, Noordwijk, The Netherlands \\ ${ }^{2} A D S$, Airbus Defense and Space, Stevenage, United Kingdom \\ ${ }^{3}$ ADS, Airbus Defense and Space, Toulouse, France \\ ${ }^{4}$ Leonardo, Pomezia, Italy \\ ${ }^{5} A D S$, Airbus Defense and Space, Rome, Italy \\ ${ }^{6} D L R$, Institute of Atmospheric Physics, Oberpfaffenhofen, Germany \\ ${ }^{7}$ ECMWF, Reading, United Kingdom \\ ${ }^{8}$ ESA-ESRIN, Frascati, Italy \\ *Email: thomas.kanitz@esa.int
}

\begin{abstract}
ESAs Earth Explorer Aeolus was launched in August 2018. Aboard the first spaceborne wind lidar ALADIN (Atmospheric LAser Doppler INstrument) was switched on in early September 2018 and demonstrated the capability to provide atmospheric wind profiles globally from particle and molecular backscatter. In doing so, it will contribute to the improvement in numerical weather prediction (NWP) and the understanding of global dynamics. At the same, it is a major step for powerful and frequency stabilized ultraviolet (UV) lasers for space applications. In parallel, ESA and its partners continue the development of this technology by setting up further ground tests based on Aeolus, and preparing the next milestone with ATLID (ATmospheric LIDar) for the Earth Cloud, Aerosol and Radiation Explorer (EarthCARE) mission. ATLID is currently fully integrated and getting prepared for its on-ground testing.
\end{abstract}

\section{INTRODUCTION}

ALADIN is the single payload of ESA's wind lidar mission Aeolus within the Earth Explorer family of the ESA Living Planet Programme [1]. Aeolus shall demonstrate the potential of spacebased Doppler Wind lidars to improve NWP and advance the understanding of atmospheric dynamics and climate processes. In order to do so, it shall provide vertical profiles of one component of the horizontal wind vector along the laser lineof-sight (LOS) from ground up to the lower stratosphere (to $30 \mathrm{~km}$ ). Aeolus was launched from Kourou (French Guiana) on 22 August 2018 and is in operation since then with only minor interruptions.

ATLID is part of the payload of the EarthCARE mission, the sixth Earth Explorer of ESA [2]. The EarthCARE payload consists of a cloud Dopper radar, an imager, and a radiometer. In a synergetic manner, the four instruments will retrieve vertical profiles of clouds and aerosols, their radiative characteristics, and their micro-physical properties in order to determine top of atmosphere radiance and flux profiles. ATLID is gearing up for its ground performance and qualification tests in 2019.

\section{AEOLUS - ALADIN}

ALADIN is a direct-detection Doppler wind lidar operating at the UV wavelength of $354.8 \mathrm{~nm}$ [3]. It is composed of a $1.5 \mathrm{~m}$-diameter telescope, a highly sensitive receiver setup with an imaging Fizeau spectrometer for narrowband Mie backscatter from aerosol and cloud particles, a double-edge Fabry-Perot spectrometer for broad Rayleigh-Brillouin molecular backscatter and photon counting accumulation charge coupled device (ACCD) detectors. The frequencystabilized UV-laser was designed to emit $80 \mathrm{~mJ}$ pulses at a repetition frequency of $50.5 \mathrm{~Hz}$. The return signal is acquired with a vertical resolution between 0.25 and $2 \mathrm{~km}$ and an accumulation of typically 20 pulses, resulting in a horizontal raw 
data resolution of $3 \mathrm{~km}$ per measurement. Further averaging (600 pulses) yields data at the so-called observation resolution which meets the wind precision requirement of 1 to $2.5 \mathrm{~m} / \mathrm{s}$ [4].

\subsection{From Launch to Operation}

Aeolus was launched on 22 August 2018. After the successful launch and early orbit phase, the commissioning was started 48 hours later. Before ALADIN could be turned on, the in-situ cleaning system (ICS) was used to purge ALADIN twice to minimize the risk of laser induced contamination (LIC). After these flushes, the ICS was set to nominal mode to operate ALADIN in a residual oxygen pressure environment starting from 31 August 2018 [5].

For the first in-orbit operations, ALADIN's transmitter Flight Model A (FM-A) was selected, rather than the most powerful and stable Model B (FM-B). FM-A switched to its warm up mode allowing the assessment of the general status of the transmitter itself, but also the thermal and power performance of the instrument, e.g., telescope and transmitter thermal regulation. In parallel the detection chain was enabled and first scientific data became available to characterize the dark signal on the ACCDs, and allowing running the data chain ground processors and dissemination facility up to Level 1 [6]. On 2 September 2018, the operation of the transmitter FM-A started in defined intermediate energy level steps. The first light through ALADINs internal path could be measured at low emit energy, followed by the first frequency scans, laser beam monitoring and spectral coregistration of the Mie and Rayleigh spectrometers. Subsequently, the first profile wind measurements were able to begin on 4 September 2018. The following unbroken four month of operation were dedicated to instrument characterization with weekly routine calibrations for, e.g., spectral characterization over different frequency ranges and special operations for targeted investigations. Within this period the existing seven instrument characterization modes were extended by three procedures. The automated mission planning facility took over in week twelve of the mission and the data was assessed fit for distribution to the Aeolus Calibration and Validation teams by the data analysis core team in mid-December 2018 $[7,8]$.

Two main instrument related issues dominated the first seven months of operation. The initial UV emit energy of FM-A was below recorded levels during last on-ground tests, respectively $65 \mathrm{~mJ}$ compared to $80 \mathrm{~mJ}$ (Fig. 1). Thermal conditions resulting in an unexpected gradient at the interface of laser and cooling systems are considered the likely root cause. During the following weeks and months, the emitted energy decreased continuously over the span of weeks with an ever increasingly shallower slope (Fig.1). Special operations including sensitivity tests of thermal adjustments of the laser cold plate temperature, and pre- and power amplifiers, as well as adjustments to the main currents were performed to keep the emit energy above $50 \mathrm{~mJ}$. This (preliminary) value was derived from NWP considerations. After a spurious switch to safe mode on 14 January, Aeolus returned to operation on 15 February 2019, still using FM-A, and a emit energy of 50mJ since 6 March 2019. No signs of LIC have been observed so far.

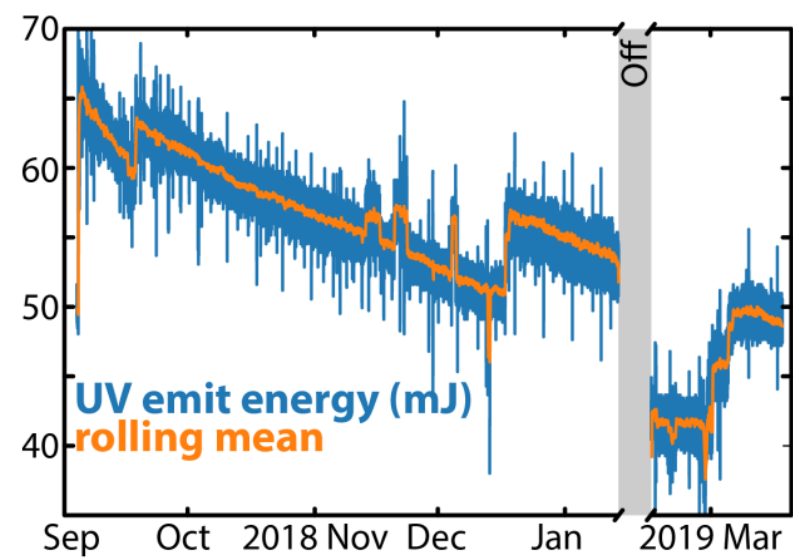

Figure 1 Aeolus inflight ultraviolet emit energy from housekeeping telemetry at power laser head level.

The second issue are appearing defects on a limited set of pixels of the ACCD (5 pixels on each channel), resulting in higher dark current rates and consequently biases in the final wind product $[7,8]$. After activation of a single pixel, the increased dark current decreases to a base offset. A dedicated calibration has been added to the routine operations and forward corrections are developed for the ground processing. Apart from these two issues, Aeolus has shown remarkable thermal and spectral stability. 


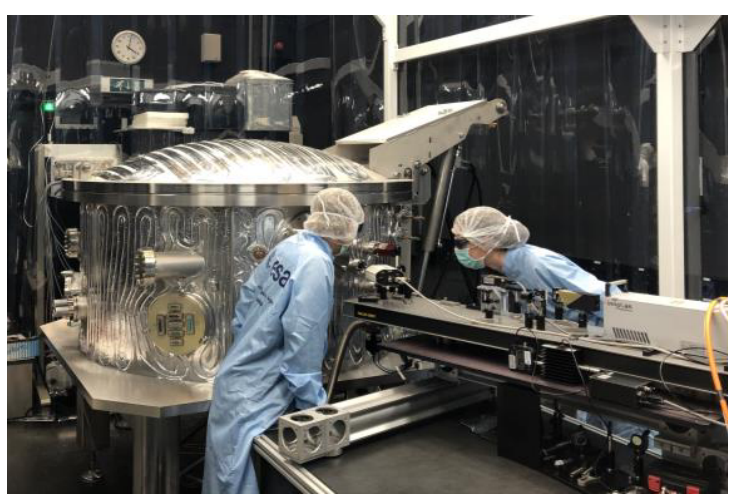

Figure 2 The Laser Operation and Verification Facility at ESTEC.

\subsection{Laser Operation and Verification Facility}

ESA is currently setting up a Laser Operation and Verification Facility at ESTEC. The aim of this facility is to verify results in laser performance induced by operational commands before ultimately applying these to the operational lasers in-flight. To achieve this goal, the Flight Model spare laser (FM-C) with equal power and reference laser heads will be operated with electrical, thermal and telemetry harness in a flight-like configuration. This includes running the laser with a low pressure oxygen supply in a vacuum chamber in order to simulate the orbital environment.

To guard against contamination inside of the vacuum chamber and on the optical ground support equipment, the major parts of the facility are installed underneath an ISO7 clean tent. The setup is placed on a seismic block to decouple the vibration sensitive measurements from environmental disturbances. Temperature stability is assured by an air condition under the tent and a shield inside the vacuum chamber. The laser will be wrapped in multi-layer insulation to minimize temperature variation that could affect the laser performance. The main outcomes of this facility are:

- Problem solving of in-flight issues by replicating the observations on ground, e.g., the impact of vibration on output performance

- The mitigation of risks associated with the change of critical laser parameter changes by applying them first on ground

- Opportunity to investigate parameters not covered by pre-flight tests and in-flight data
The operation of this facility will help to improve the knowledge of high UV energy lasers with demanding frequency stability requirements and their operation in the space for future missions.

\subsection{Next step for In-Flight Monitoring Sensors}

In-Flight Monitoring Sensors (IFM) implemented in ALADIN and ATLID flight model lasers were limited to measuring temperatures and energies. Lessons learnt from on-ground testing and inflight operation indicate the need of more extensive monitoring, e.g., the spatial beam characteristics (beam near field profiles, fluence, divergence, and pointing) and pulse duration.

Leonardo is currently developing a set of innovative and more sophisticated sensors for future spaceborne applications. Each sensor is mainly composed of a classical power and a new imaging section in order to fulfill the identified needs.

A complementary metal-oxide-semiconductor (CMOS) material was selected for the imaging sensor. The challenge here is to realize a detector working at both 532 and $355 \mathrm{~nm}$ and possessing at least 0.5 MPixels, a $40 \%$ active area filling-factor and a $5 \mu \mathrm{m}$ pixel-size size while being constrained to $50 \times 50 \times 30 \mathrm{~mm}$ interface cube size.

For the energy section the photodiodes already customized for ALADIN/ATLID lasers were selected, where the light is scattered with a diffusing ceramic element on a photodiode.

In order to reduce the signal sensitivity, the beam impinging on the photodiodes can be scattered by an integrating sphere achieving total collection of light, spatial integration, and insensitivity to beam motions.

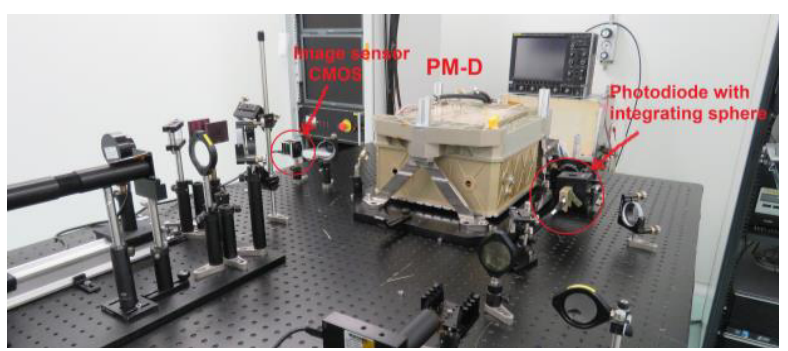

Figure 3 The IFM sensors (image and energy) bread boarding with PM-D at Leonardo.

The characterization of the IFM sensors will be done on the Proto Model (PM-D), a laser based on 
all rejected parts from ALADIN FMs. based on rejected parts from ALADIN FMs. Despite its origins, the laser performs at a high level. It emits more than $110 \mathrm{~mJ}$ UV emit energy, beam dimensions of $5.4 \times 4.4 \mathrm{~mm}$, divergence of $270 \times 660 \mu \mathrm{rad}$, and pulse width of $21.5 \mathrm{~ns}$.

\section{EarthCARE - ATLID}

ATLID's task is to provide vertical profiles of optical properties of thin cloud and aerosol layers, as well as the altitude of cloud boundaries. ATLID includes a laser similar to ALADIN [9], but pressurized and with only one pump unit. It emits laser pulses with $40 \mathrm{~mJ}$ energy in the UV, at a repetition rate of $51 \mathrm{~Hz}$. The backscatter signal is collected by a $620 \mathrm{~mm}$ telescope, and is then filtered and redirected through the optical focal plane assembly, allowing independent measure of atmospheric Mie and Rayleigh return signals.

Following the manufacturing, qualification and delivery of all ATLID units, the optical and electrical integration has been conducted in parallel in order to assemble the Optical Flight Model and the Electrical Flight Model. Following the initial performance and functional tests of these models [10], and with the integration of the flight laser cooling system, the instrument assembly approaches its final flight configuration. After the completion of additional instrument functional verification tests, the full instrument performance and qualification test campaigns are planned to start second quarter 2019 , followed by the instrument delivery to the satellite prime.

\section{CONCLUSIONS}

ESAs lidar missions with ALADIN and ATLID have had a long road of development. ALADIN is technological pioneer for powerful frequencystable UV lasers in space. The launch of ALADIN and its successful operation covering several months to date has demonstrated the potential of space-borne lidars to deliver wind profiles and other important geophysical parameters. ESA, together with its partners, are establishing a valuable ground test facility to further improve the technological knowledge, which adds to the experience of inflight data and will be useful for ATLID operation in the future, too.

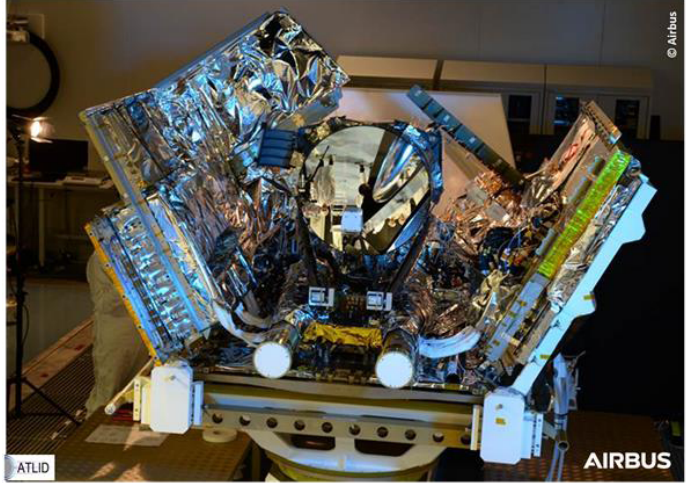

Figure 4 Optical Flight Model inside the housing structure of the Electrical Flight Model.

\section{ACKNOWLEDGEMENTS}

ESA wants to thank the science community and its partners for the continuous support during the development of these challenging Earth Explorers.

\section{REFERENCES}

[1] ESA, 2008: ADM-Aeolus Science Report, ESA report SP-1311, $121 \mathrm{pp}$.

[2] http://esamultimedia.esa.int/docs/SP_1279_1_Ear thCARE.pdf

[3] Morancais, D., et al., 2004: ALADIN: The first European lidar in space. Proceedings of the $22^{\text {nd }}$ ILRC

[4] European Space Agency ESA, 2016: ADMAeolus Mission Requirements Documents, AERP-ESA-SY-001, Issue 2, 16/11/2016, 57pp.

[5] Kanitz, T., et al., 2018: Aeolus First Light - First Glimpse. Proc. Int. Conf. Space Optics, ICSO.

[6] Reitebuch, O., Huber, D., Nikolaus, I., 2018: Algorithm Theoretical Basis Document ATBD: ADM-Aeolus Level 1B Products, AE-RP-DLRL1B-001, V. 4.4, 20.04.2018, 117pp.

[7] Reitebuch, O. et al., 2019: Initial assessment of the performance of the first wind lidar in space on Aeolus, Proceedings of the $29^{\text {th }}$ ILRC.

[8] Straume, A.G. et al., 2019: ESA'S space based Doppler mission Aeolus - First Results of the Wind and Aerosol Product Assessment, Proceedings of the $29^{\text {th }}$ ILRC.

[9] Cosentino, A., et al., 2012: Spaceborne Lasers Development for ALADIN and ATLID Instruments, Geoscience and Remote Sensing Symposium, IGARSS.

[10] de Villele, G. et al., 2018: ATLID, ESA Atmospheric Lidar: integration of instrument and tests, Proc. Int. Conf. Space Optics, ICSO. 